\title{
Treatment and prognosis of pleuropulmonary blastoma: A Single-Center Report of 31 Cases
}

\author{
Feng Wang ${ }^{1}$, Lin Zhuang ${ }^{2}$, Jianghua Liang ${ }^{1}, \mathrm{Le} \mathrm{Li}^{1}, \mathrm{Hui} \mathrm{Wang}^{2}$, Jue Tang${ }^{1}$, Jia Zeng ${ }^{1}$, and \\ Wei Liu ${ }^{1}$ \\ ${ }^{1}$ Guangzhou Women and Children's Medical Center \\ ${ }^{2}$ Guangzhou Women and Children's Medical Center
}

September 4, 2021

\begin{abstract}
Objectives: Pleuropulmonary blastoma (PPB) is a very rare, characteristic and highly aggressive neoplasm occurring in children, most under 6 years of age. We assessed the clinical characteristics, treatment modalities, treatment outcomes, and prognostic factors affecting survival in patients with PPB treated at our institution over a 10-year period to improve the prognosis of PPB. Methods: From November 2008 to November 2019, 31 children (21 boys and 10 girls) with a median age of 30 months (range, 22 days-54 months) were treated at our institution. Here, we describe the patient characteristics, treatment modalities and treatment outcomes. The Kaplan-Meier method was used to estimate the progression free survival probability (PFS) and overall survival (OS). Log-rank test was performed for comparison between groups. Results: 3 children were lost to follow-up and 2 were dead of postoperative complications. Of the 26 patients included in the follow-up, 16 PPB patients displayed tumor-free survival. The 6-month, 1-, 3-, and 5-year PFS were 80.8\%, 69.0\%, 60.4\% and 60.4\%, respectively. Accordingly, the 6-month, 1-, 3-, and 5-year OS were $84.6 \%, 72.7 \%, 60.1 \%$ and $60.1 \%$, respectively. Sex, extent of surgery and chemotherapy/irradiation appeared to affect the survival, while age and pathology type appeared not to do. Conclusions: PPB is an aggressive neoplasm. To improve the prognosis of $\mathrm{PPB}$, we should promote radical resection and improve the auxiliary treatment measures.
\end{abstract}

\section{Introduction}

Pleuropulmonary blastoma (PPB) is a very rare, highly aggressive neoplasm occurring in the lung or pleura and presenting in children, most under 6 years of age. It was initially proposed to be a distinct entity in 1988 as a dysembryonic malignancy. ${ }^{1} \mathrm{PPB}$ is histologically characterized by primitive blastema and a malignant mesenchymal stroma that often shows multidirectional differentiation (rhabdomyosarcomatous, chondrosarcomatous, liposarcomatous, etc.). The tumor is usually located in the lung periphery, but may be extrapulmonary, involving the parietal pleura, mediastinum and diaphragm. Despite the introduction of multimodal therapy, the overall prognosis for PPB patients remains poor. Venkatramani et $\mathrm{al}^{2}$ studied a series of $12 \mathrm{PPB}$ cases and found that the 5 -year overall survival was $42 \%$. In this report, we retrospectively summarized and analyzed the clinical characteristics and living conditions in children with PPB who were admitted to our medical center from November 2008 to November 2019.

\section{Patients and methods}

The study have been approved by the Committee on Human Research at our medical center. PPB cases were included if the pathology confirmed PPB by two different pathologists. The PPB cases included in this report were diagnosed from November 2008 to November 2019 at our medical center. Altogether, 31 children were pathological diagnosis of pleuropulmonary blastoma. All of the 31 children underwent CT and chest radiography, 8 underwent echocardiography, 3 underwent thoracic ultrasonography. However, only 15 children were diagnosed with pleuropulmonary blastoma before operation. As for others, 6 were diagnosed 
with lung cyst, 6 with other thoracic tumors, 2 were diagnosed with encapsulated effusion and 2 with chest wall tumor. Data of the 31 children were abstracted from medical records after participant-informed consent. SAS9.4 was used for statistical analysis. More concretely, count data were described by the number and rate of cases. Comparison between groups was performed using Fisher's exact test and Kaplan-Meier method to calculate the cumulative survival rate and draw the survival curve. Log-rank test was performed for comparison between groups. Bilateral $\mathrm{P}<0.05$ was considered statistically significant.

\section{Results}

\subsection{Clinical features}

Of the thirty-one children included in this analysis, 21 were male and 10 were female. The youngest patient was 22 days at the time of diagnosis and the oldest was 70 months (median age, 30 months). Presenting symptoms included respiratory distress in 23 patients, cough in 22, fever in 12, poor appetite in 5, chest pain in 3, abdominal pain in 2, chest wall mass in 1, hemoptysis in 1 and facial swelling in 1 . Six of the 31 patients were admitted to the pediatric intensive care unit (PICU) because of their critical condition. Four patients were previously diagnosed with pulmonary cystic lesions and another 3 patients had a history of pneumothorax.

Histology was type I PPB in 5 (no type Ir PPB), type II in 5, and type III in 21 patients. Fourteen patients had primary tumors in the left lung and 15 in the right lung, while 2 patients had their primary tumors in the chest wall. Of the 31 patients, 15 patients had extrapulmonary involvement (mediastinum, parietal pleura, and diaphragm) and no one had any evidence of tumor metastasis.

\subsection{Treatment}

The extent of surgical resection was estimated after detailed review of surgical notes as follows: 1) biopsy; 2) partial resection, when a macroscopic tumor remained; and 3) macroscopic total resection, when the surgeon reported a total resection. Upfront surgical resection was attempted in 30 patients and macroscopic total resection was achieved in 19 patients, while the remaining one tumor in the chest wall was considered unresectable and only a biopsy was performed.

Chemotherapy with IVADo (vincristine $1.5 \mathrm{mg} / \mathrm{m}^{2}$, D1; actinomycin D $1.5 \mathrm{mg} / \mathrm{m}^{2}$, D1; Theprubicin $30 \mathrm{mg} / \mathrm{m}^{2}$, D1-2 and ifosfamide $\left.3 \mathrm{~g} / \mathrm{m}^{2}, \mathrm{D} 1-2\right)$ and irradiation (30Gy $\left.\sim 36 \mathrm{~Gy}\right)$ were recommended for all type II/III patients after operation. However, only 17 patients received chemotherapy and irradiation.

\subsection{Outcome}

Three patients were lost during follow-up. One type III PPB patient died of pneumonia and another died of heart failure just after the operation. At last, 26 patients were included in the follow-up. The shortest follow-up time was 1 month and the longest was 136 months. The median follow-up time was 29.5 months.

A type III PPB patient succumbed one month later because of an intra-atrial tumor thrombus after the operation. A patient who was diagnosed with type III PPB by biopsy gave up on treatment and succumbed to PPB 20 days later. Five patients had a relapse 2 to 18 months after the operation, 4 of whom received chemotherapy and irradiation after the operation and 1 who did not. Two patients who received chemotherapy and irradiation had a bone/brain metastasis 9 to 11 months after the operation, while 1 without chemotherapy/irradiation suffered from bone/renal metastasis within 3 months. All 5 patients with tumor relapse underwent a second operation; however, it was difficult to perform macroscopic total resection and they suffered disease progression soon after and succumbed within 2 to 6 months after the second operation. The 3 patients with tumor metastasis gave up on treatment and succumbed to PPB 1 to 4 months later. The other 16 PPB patients displayed tumor-free survival. Among the 16 patients, 2 suffered from cystic lung lesions at 1 and 3 months respectively after completing primary treatment. Neither of these patients received further treatment and they remained stable at 93 or 120 months after the initial diagnosis.

On the whole, a total of 10 children died and the other 16 survived. The comparison of baseline characteristics between the dead and the alive children is shown in Table 1. By calculating,the 6-month, 1-, 3-, and 5-year 
progression free survival probability (PFS) were $80.8 \%, 69.0 \%, 60.4 \%$ and $60.4 \%$, respectively.(Table 2) Two years is a rapid period of disease progression. The PFS of girls was higher than that of boys and of children who accepted chemotherapy/irradiation was higher than that of children who didn't $(\mathrm{P}<0.05)$.Furthermore, the PFS of children with complete tumor resection was higher than that of children with partial tumor resection (the difference was statistically significant on the margin, $\mathrm{P}=0.077$ ). However, age and pathology type did not affect the PFS (Fig 1).

Accordingly, the 6-month, 1-, 3-, and 5-year overall survival probability (OS) were $84.6 \%, 72.7 \%, 60.1 \%$ and $60.1 \%$,respectively.(Table 2) Two years is a rapid period of death. The OS of girls was higher than that of boys and of children who accepted chemotherapy/irradiation was higher than that of children who didn't $(\mathrm{P}<0.05)$.Furthermore, the OS of children with complete tumor resection was higher than that of children with partial tumor resection (the difference was statistically significant on the margin, $\mathrm{P}=0.073$ ). However, age and pathology type did not affect the OS (Fig 2).

\section{Discussion}

PPB is a characteristic tumor in children. Its histology can manifest as cystic, substantial or mixed. Accordingly, Dehner et al classified PPB into three types as follows: Type I, tumor composed of cystic elements; Type II, tumor composed of both cystic and substantial elements; and Type III, tumor composed of substantial elements. ${ }^{3}$ The three PPB pathologies form a dynamic continuum. While Type I PPB tends to be a low-grade malignancy, it can progress to the more malignant type II or III. Three patients in the present study had a history of pulmonary cystic lesions, and when they were operated on, the tumor filled the thorax. Two of the 3 patients suffered from tumor relapse soon after the operation, while the other patient was lost during follow-up. We can deduce that the cystic lesions in the lungs of the 3 patients are type I PPB. If the three patients had been operated on when they were diagnosed with pulmonary cystic lesions, their results would have been much better. It is difficult to distinguish type I PPB from a benign cyst by radiography. Thus, we suggest resecting asymptomatic lung cysts in children.

It has been reported that the prognosis of PPB is related to the pathological type. While the prognosis for type I PPB is good, the prognosis for type II and type III PPB is very poor. Messinger et $\mathrm{al}^{4}$, who are members of the International Pleuropulmonary Blastoma Registry (IPBR), studied a series of 350 PPB cases and calculated the 5-year overall survival rate to be $91 \%$ for type I, $71 \%$ for type II, and $53 \%$ for type III patients. Bisogno et $\mathrm{al}^{5}$, who are members of the European Cooperative Study Group for Paediatric Rare Tumours (EXPeRT), studied a series of 65 PPB cases and found that the overall survival was $91.7 \%$ for type I patients and $57.5 \%$ for type II and type III patients. In the present study, all 5 type I PPB patients are alive without tumors, with the longest survival time as 136 months. By calculation, the PFS at 5 year for type I PPB is $100 \%$, but for type II PPB is $75 \%$ and for type III PPB is $43.7 \%$. However, there was no significant difference in survival curve among the three pathological types. It may be related to the uneven and small number of specimens.

Except the pathological type, the present study found that the extent of surgery and chemotherapy combined with irradiation appeared to be related to PPB prognosis. If the tumor can be macroscopically completely resected and the PPB patients accept chemotherapy as well as irradiation in addition to the operation, the results would be better. What interesting is that, girls in the study seem to have a better prognosis than boys, which has not been reported before. Why? The question needs further study. Several other studies also studied the factors related to PPB prognosis. Invasiveness, complete tumor resection, type I PPB, chemotherapy and tumor size were the main factors. ${ }^{5,6}$ To achieve better results for PPB patients, we need to strive for complete tumor removal combined with chemotherapy. Unfortunately, PPB progresses rapidly such that when patients arrive at the hospital, most of the tumors are too large to be completely resected. For example, in the present series, of the 26 type II/III PPB patients, 12 had large tumors invading vital structures that resulted in incomplete resection. Therefore, some specialists suggest that patients with unresectable tumors be initially treated with core needle biopsy and neoadjuvant chemotherapy to reduce the tumor size and make it resectable. ${ }^{7-10}$ Following the protocol, the five-year overall survival was elevated from $44 \%$ to $68 \% .{ }^{11}$ However, core needle biopsy cannot always yield the correct diagnosis because it may 
penetrate necrotic tissue. Furthermore, PPB progresses very rapidly and patients may not be able to wait for pathological results; for example, Xi Chai described how four of six patients developed adverse events while waiting for pathological results after core needle biopsy. ${ }^{12}$ For these reasons, we have not adopted core needle biopsy, rather upfront excision.

PPB is sensitive to chemotherapy, but there is no standard regimen. In 2007, following communication with sarcoma experts in Europe and North America, the IPBR recommended that chemotherapy for type II/III PPB consist of IVADo and that it consist of VAC (vincristine, actinomycin D, and cyclophosphamide) for type I. ${ }^{13}$ We adopted the recommendation for type II/III PPB, similar to many other medical centers, 14,15 and we have proven it to be effective. It should be noted that patients in the present series received irradiation in addition to chemotherapy. However, we cannot prove the effectiveness of chemotherapy alone or chemotherapy combined with irradiation. For type I PPB, some experts believe it can be cured by radical resection alone and that chemotherapy does not improve its prognosis ${ }^{4,16}$. Consistently, 5 type I PPB patients in the present study did not receive chemotherapy or irradiation after the operation. None of them suffered from tumor relapse or metastasis during the follow-up period. It appears that operation is enough for type I PPB. Of course, rigorous surveillance after the operation is needed for early detection of recurrence.

Metastases occur in up to $30 \%$ of patients with type II/III PPB, and the most common metastatic site is the brain ${ }^{17,18}$. Inconsistently, there were only 3 metastases in the present study, though relapse was more common. If patients suffer from tumor relapse or metastasis, the outcome is generally very bad because we have no effective treatment for progressive tumors. Some experts have introduced new treatments for progressive PPB via their experiences. Behery et $\mathrm{al}^{19}$ reported a PPB case with brain metastasis that was treated with surgery followed by chemotherapy and irradiation. After that, the patient underwent high-dose chemotherapy and blood stem cell transplantation. Since completing these therapies, the patient has done well without any evidence of tumors. Ohta et al ${ }^{20}$ adopted a protocol with irinotecan and vincristine to treat a PPB patient with lung metastases. After the third course, the metastases disappeared and there was no recurrence. Nakano et $\mathrm{al}^{21}$ described two PPB cases with brain metastasis that were treated with gross total resection of the

metastatic tumors. Afterwards, one patient received 1 course of CDDP/CPT-11 (cisplatin and irinotecan), 2 courses of TMZ/ETP (temozolomide, and etoposide) and 6 courses of intrathecal methotrexate while undergoing whole-brain radiation. The other case received radiotherapy to the tumor bed followed by one course of ICE (ifosfamide, carboplatin, and etoposide) and an antiangiogenic multidrug regimen over a 22month period. Both of the patients are currently alive and in complete remission. These methods appear effective to treat progressive PPB. However, these are just case reports. More effective treatments for recurrent and metastatic $\mathrm{PPB}$ are still under investigation.

In conclusion, the overall survival for type II/III patients is still low, leading us to wonder how to improve the prognosis of PPB. The problem can be dissected into determining how to completely resect the tumor and improve the auxiliary treatment measures. Prospective large sample studies are needed to achieve this goal.

Declarations of interest: none.

\section{References}

Christosova IR, Avramova BE, Drebov RS, Shivachev HI, Kamenova MA,

Bobev DG,Brankov OG. Diagnosis and treatment of pleuropulmonary blastoma-single center experience. Pediatr Pulm 2015; 50(7):698-703. DOI:10.1002/ppul.23047.

2. Venkatramani R, Malogolowkin MH, Wang L, Mascarenhas L. Pleuropulmonary Blastoma: A Singleinstitution Experience. J Pediatr Hematol Oncol 2012; 34:e182-5. DOI:10.1097/MPH.0b013e3182546adf.

3. Romeo C,Impellizzeri P,Grosso M, Vitarelli E,Gentile C. Pleuropulmonary blastoma: long-term survival and literature review. Med Pediatr Oncol 1999; 33(4):372-6. DOI:10.1002/(SICI)1096- 
911X(199910)33:43.0.CO;2-E.

4. Messinger YH, Stewart DR, Priest JR, Williams GM, Harris AK, Schultz KAP, Yang JD, Doros L, Rosenberg PS, Hill DA, et al. Pleuropulmonary Blastoma: A Report on 350 Central Pathology-Confirmed Pleuropulmonary Blastoma Cases by the International Pleuropulmonary Blastoma Registry. Cancer 2015; 121(2):276-85. DOI:10.1002/cncr.29032.

5. Bisogno G, Brennan B, Orbach D, Stachowicz-Stencel D,Cecchetto T, Indolfi G, Bien P,Ferrari E,Dommange-Romero A, Florence. Treatment and prognostic factors in pleuropulmonary blastoma: An EXPeRT report. Eur J Cancer 2014; 50(1):178-84. DOI:10.1016/j.ejca.2013.08.015.

6. Choudhury M, Gupta SK, Jain M, Chadha R. Pleuropulmonary Blastoma With Cervical Lymph Node Metastasis at Presentation. Indian Pediatr 2011; 48:146-7. DOI:10.1007/s12098-010-0252-5.

7. Çorapçıŏlu F, Liman T, Aksu G, Inan N,Topçu S. A case report with type II pleuropulmonary blastoma:successful treatment with surgery and chemotherapy. Turkish J Pediatr 2009; 51: 78-81. DOI:doi:10.3109/01942630903246026.

8. Addanki A,Chaitanya K,Bartakke S, Srividya, Sethuratnam. A Case Report of Pleuropulmonary Blastoma Presenting as Tension Pneumothorax. Indian J Med Paediatr Oncol 2017; 38(1):70-2. DOI: 10.4103/09715851.203515.

9. Fita AM, Llinares-Riestra E, Domenech-Abellan E, Bermudez-Cortes M , Galera-Minarro AM , BasBernal A, Fuster-Soler JL . Type III pleuropulmonary blastoma in a dicer1 germline mutation carrier. The management of residual lung cystic lesions. Pediatr Blood Cancer 2017; 00:e26438. DOI:10.1002/pbc.26438.

10. Parsons SK, Fishman SJ, Hoorntje LE, Jaramillo D, Marcus KC, Perez-Atayde AR, Kozakewich HP, Grier HE, Shamberger RC. Aggressive Multimodal Treatment of Pleuropulmonary Blastoma. Ann Thorac Surg 2001; 72:939-42. DOI:10.1016/S0003-4975(00)02411-5.

11. Sparber-Sauer M, Seitz G, Kirsch S, Vokuhl C, Leuschner I, Dantonello TM, Scheer M, Kalle T , Ljungman G , Bielack SS, et al. The impact of local control in the treatment of type II/III pleuropulmonary blastoma. Experience of the Cooperative Weichteilsarkom Studiengruppe (CWS). J Surg Oncol 2017; 115(2):164-72. DOI:10.1002/jso.24416.

12. Chai X, Zhang N, Zhang DW, Fu LB, Yu T, Su Y, Gao HM, Qian SY, Zeng Q, Ma XL. Clinical analysis of early death in children with pleuropulmonary blastoma in a single center in China. Pediatr Blood Cancer 2019; e27802. DOI:10.1002/pbc.27802.

13.Fosdal MB. Pleuropulmonary Blastoma. J Pediatr Oncol Nurs 2008; 25(6):295-302. DOI:10.1177/1043454208323292.

14. Pierce JM, Lacroix P, Heym K, Bowman WP, Margraf L, Iglesias J, Ray A. Pleuropulmonary Blastoma: A Single-center Case Series of 6 Patients. J Pediatr Hematol Oncol 2017; 39(8):e419-22. DOI:10.1097/MPH.0000000000000972.

15. Indolfi P, Casale F, Carli M, Bisogno G, Ninfo V, Cecchetto G, Bagnulo S, Santoro N, Giuliano M, Tullio MTD. Pleuropulmonary Blastoma. Cancer 2000; 89(6):1396-401. DOI:10.1002/10970142(20000915)89:63.0.CO;2-2.

16. Zhang N, Zeng Q, Ma X, Chen CH, Yu J, Zhang X, Yan D , Xu CQ, Liu DY, Zhang Q. Diagnosis and Treatment of Pleuropulmonary Blastoma in Children: A Single-Center Report of 41 Cases. J Pediatr Surg 2020; 55(7):1351-55.DOI: 10.1016/j.jpedsurg.2019.06.009.

17. Miniatib DN, Chintagumpalac M, Langston C, Dishop MK, Olutoye OO, Nuchtern JG, Cass DL. Prenatal presentation and outcome of children with pleuropulmonary blastoma. J Pediatr Surg 2006; 41, 66 - 71. DOI:10.1016/j.jpedsurg.2005.10.074. 
18. Priest JR, Magnuson J, Williams GM, Abromowitch M, Byrd R, Sprinz P, Finkelstein M, Moertel CL, Hill DA. Cerebral metastasis and other central nervous system complications of pleuropulmonary blastoma. Pediatr Blood Cancer 2007; 49: 266-73.DOI: https://doi.org/10.1002/pbc.20937.

19. Behery RE, Bedrnicek J, Lazenby A, Nelson M, Grove J, Huang D, Smith R, Bridge JA. Translocation t $(12 ; 17)$ (q24.1; q21) as the Sole Anomaly in a Nasal Chondromesenchymal Hamartoma Arising in a Patient with Pleuropulmonary Blastoma. Pediatr Devel Pathol 2012; 15(3):249-53. DOI:10.2350/11-11-1121-CR.1.

20. Ohta Y, Fujishima M, Hasegawa H, Kosumi T, Yonekura T. High Therapeutic Effectiveness of Postoperative Irinotecan Chemotherapy in a Typical Case of Radiographically and Pathologically Diagnosed Pleuropulmonary Blastoma. J Pediat Hematol Onc 2009; 31(5):355-8. DOI:10.1530/eje.1.02313.

21. Nakano Y, Gatell SP, Schultz KAP, Carrillo TM, Fujisaki H, Okada K, Horiike M, Nakamura T, Watanabe Y, Matsusaka Y, et al. Successful treatment of metastatic cerebral recurrence of pleuropulmonary blastoma. Pediatr Blood Cancer 2019; e27628. DOI:10.1002/pbc.27628.

Fig1 Progression free survival curve of children with different characteristics. (*Total sample size: 21)

Fig2 Overall survival curve of children with different characteristics. (*Total sample size: 21 )

\section{Hosted file}

Table1.docx available at https://authorea.com/users/432790/articles/536256-treatment-andprognosis-of-pleuropulmonary-blastoma-a-single-center-report-of-31-cases

\section{Hosted file}

Table2.docx available at https://authorea.com/users/432790/articles/536256-treatment-andprognosis-of-pleuropulmonary-blastoma-a-single-center-report-of-31-cases 

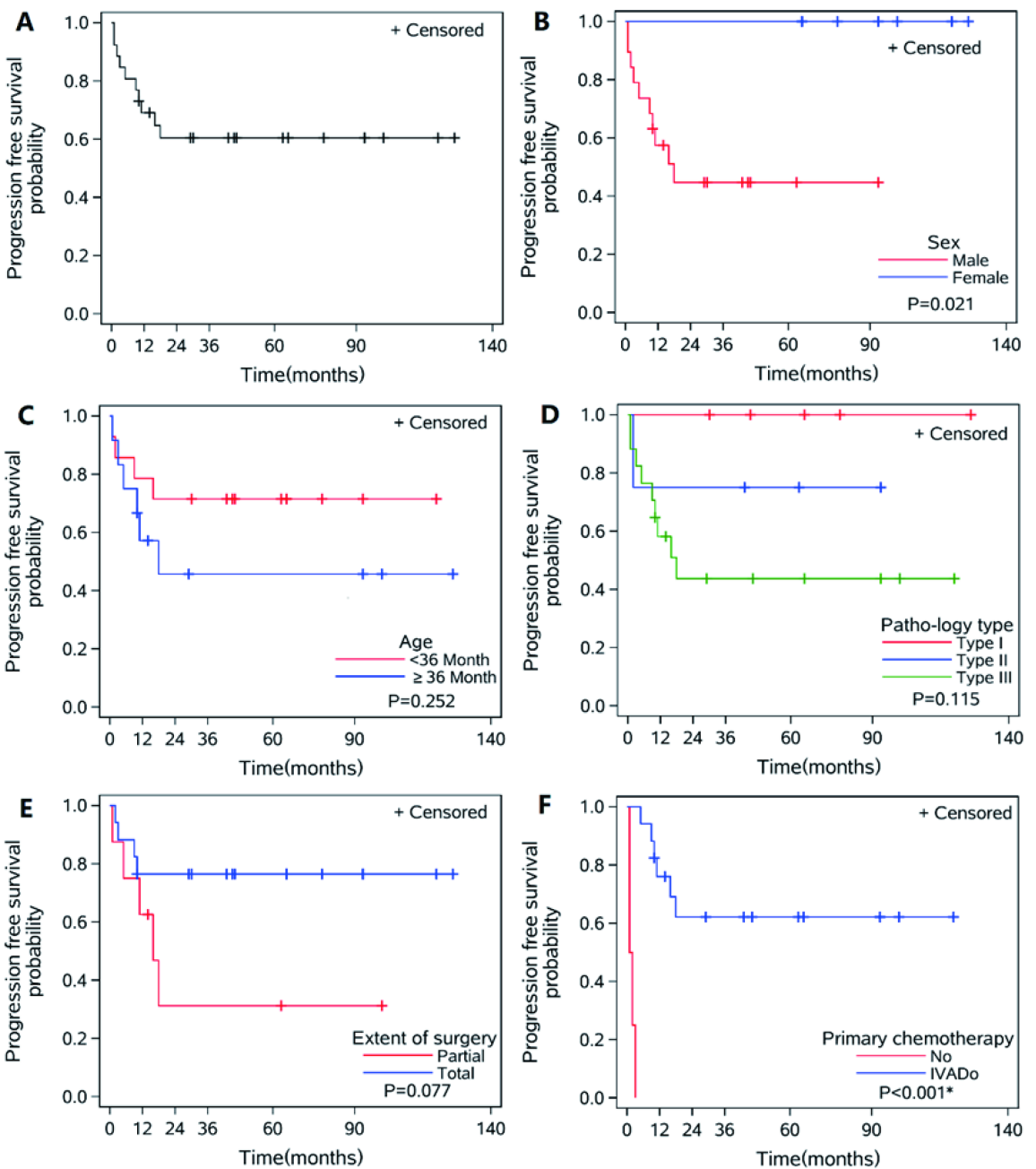

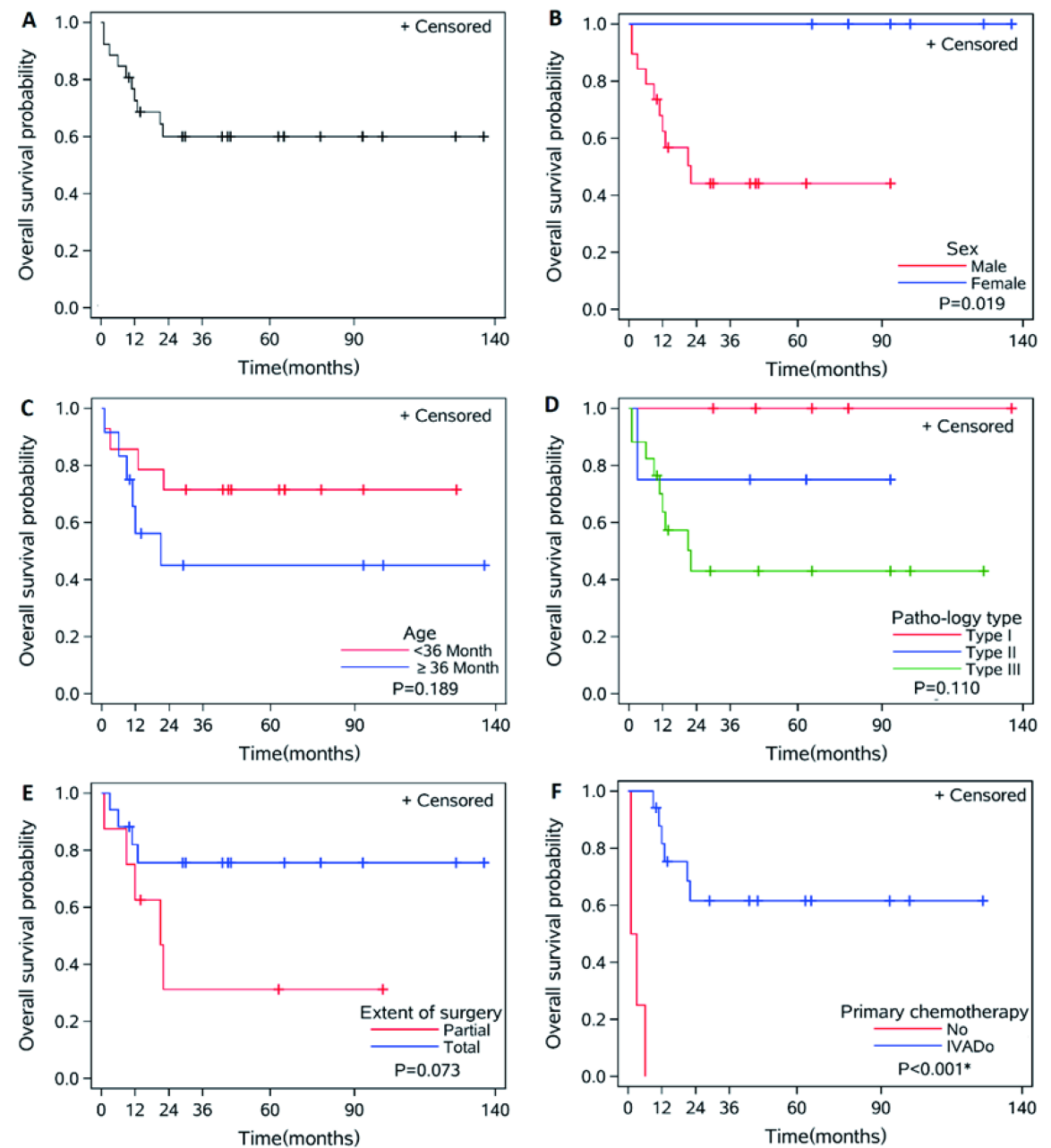